\title{
Unusual solvent effects in the reactions of 1-halo-2,4- dinitrobenzenes and aniline in aprotic and dipolar-aprotic solvents. Effects of aggregates
}

\author{
Cecilia E. Silvana Alvaro ${ }^{a}$ and Norma Sbarbati Nudelman ${ }^{b, c^{*}}$ \\ ${ }^{a}$ Departamento de Química. Facultad de Ingeniería. Universidad Nacional del Comahue. \\ Buenos Aires 1400. (8300) Neuquén. Argentina. ${ }^{b}$ Depto Química Orgánica. Facultad de \\ Ciencias Exactas. Universidad de Buenos Aires. Pab. II, Cdad. Universitaria 1428 Buenos Aires \\ Argentina. ' Institute for Materials Chemistry and Engineering, Kyushu University, 6-10-1, \\ Fukuoka, 812-8581 Japan \\ E-mail:nudelman@qo.fcen.uba.ar,nudelman@ms.ifoc.kyushu-u.ac.jp \\ Dedicated to Prof. Edmundo Rúveda and to Prof. Roberto A. Rossi \\ (received 23 Jun 03; accepted 05 Aug 03; published on the web 07 Aug 03)
}

\begin{abstract}
The kinetics of reactions between 1-halogeno-2,4-dinitrobenzene (halogen $=\mathrm{F}, \mathrm{Cl}$ ) and aniline were studied in toluene, acetone, dimethylsulfoxide and in toluene-acetone mixtures. Unexpectedly, when chlorine is the nucleofugue, the second rate coefficients, $k_{\mathrm{A}}$, in toluene shows a parabolic dependence on the amine concentration which indicates a third order in amine kinetic law, while in the dipolar aprotic solvent the dependence is linear. Small additions of acetone to the solvent produce an important increase in rate, much greater then the increase in the permittivity, which is surprising on the basis of the classical effect of a dipolar medium on a dipolar transition state. The reactions with fluorine as the nucleofugue show similar behaviours. All these results are well explained in the frame of the "dimer nucleophile" mechanism.
\end{abstract}

Keywords: Aromatic nucleophilic substitutions, aprotic solvents, mixed aggregates, dimer nucleophile mechanism

\section{Introduction}

Aromatic nucleophilic substitutions $\left(\mathrm{S}_{\mathrm{N}} \mathrm{Ar}\right.$ ) by amines have been recognized to be extremely affected by the solvent. ${ }^{1,2,3}$ Their study is currently receiving increased interest since they constitute a suitable model to contribute to the understanding of the microscopic properties of solvent in reactions due the complex interactions between the amine, the substrate and/or the intermediate that occur involving the solvent molecules. ${ }^{4,5,6}$ The mechanisms of reactions in 
protic solvents are well established1 but are the $\mathrm{S}_{\mathrm{N}} \mathrm{Ar}$ in aprotic solvents those that exhibit most peculiar features to study specific and non-specific solvent effects. ${ }^{2}$ Evidences for nucleophile aggregation, $^{7}$ substrate-nucleophile EDA complexes ${ }^{8}$ and other type of complexes ${ }^{9,10}$ have been reported by different authors when reactions were carried out in aprotic solvents. On the other hand, several attempts to establish empirical scale of microscopic parameters able to provide a quantitative description of solvent characteristic have been currently reported. ${ }^{11,12,13}$

Most of the previous studies on solvent effects in $\mathrm{S}_{\mathrm{N}} \mathrm{Ar}$ have been performed in pure solvents. ${ }^{1,2}$. Nevertheless, different studies aimed at the characterization of mixed solvents and the determination of the kinetic data of $\mathrm{S}_{\mathrm{N}} \mathrm{Ar}$ reactions in binary mixtures have been recently reported. ${ }^{4,14}$ Likewise, studies in solvent mixtures, which were held taking into account their hydrogen bond donor (HBD) or hydrogen bond acceptor (HBA) characteristics, were performed and discussed.

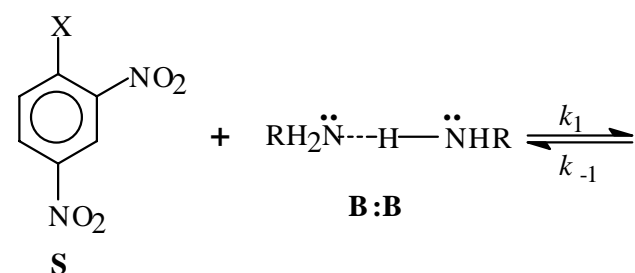

$\mathrm{S}$
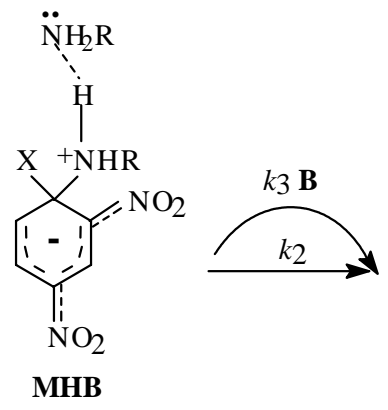

MHB

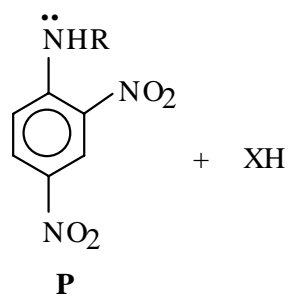

\section{Scheme 1}

We have recently published unusual findings in $S_{N} A r$ carried out in aprotic solvents. Most of these "abnormal" results are characterized, among other features, by a third-order dependence on [amine] and can be consistently explained by a mechanism called the "dimer nucleophile mechanism". ${ }^{7}$ In that mechanism, the dimer of the amine acts as a nucleophile forming an intermediate complex, a third molecule of amine assists the decomposition step (scheme 1). The intermediate is highly zwitterionic and the extra amine molecule should help to stabilize the developing charge in nonpolar aprotic solvents. The reaction with the monomer nucleophile is also possible, but the reaction with the dimer is faster because of its higher donicity. ${ }^{15,16}$

We have previously reported SNAr carried out in binary solvents such toluene-octanol, ${ }^{17 a}$ toluene-methanol, ${ }^{17 b}$ benzene-n-hexane and toluene-tetrahydrofuran, ${ }^{4 a}$ the observed specific solvent effects could be satisfactorily interpreted by the "dimer nucleophile mechanism". ${ }^{7}$ The reactions of 2,4-dinitrochlorobenzene ( $\mathrm{DNClB}$ ) with amines are currently been considered as occurring by the widely accepted two-step mechanism with the first step being rate determining, but the results obtained in the present study cannot be interpreted by that mechanism. The present work describes the study of the reactions of 2,4-dinitrochlorobenzene (DNClB) and 2,4dinitrofluorbenzene (DNFB) with aniline in toluene, acetone (AC), dimethylsulphoxide (DMSO) and in binary toluene-AC, mixtures. The observed results afford evidence concerning the critical 
role that aggregation of the nucleophile and formation of “mixed aggregates" plays in the kinetic behaviour of these reactions.

\section{Results and Discussion}

In a thorough examination of the solvents effects in aprotic and dipolar aprotic solvents, the reactions of DNClB and of DNFB with aniline in toluene, ${ }^{4 a}$ acetone, DMSO and acetone-toluene binary solvents the rate dependence with amine concentration was studied. The reactions proceed straightforwardly to give the expected N-substituted-2,4-dinitroaniline, a quantitative yield of the substitution product was obtained in all the reactions under study and pseudo-first-first order kinetics were observed throughout the work

\section{Reaction of DNCIB and DNFB with aniline in pure aprotic and dipolar aprotic solvents}

The kinetics of the reactions of aniline with 2,4-dinitrochlorobenzene, DNClB, and 2,4dinitrofluorobenzene, DNFB, in toluene and acetone at $40^{\circ} \mathrm{C}$ and DMSO at $30^{\circ} \mathrm{C}$ in the presence of variable amounts of the nucleophile were studied. Tables 1 and 2 shows the $k_{A}$ values for the reactions in toluene, acetone and DMSO, for DNCIB and DNFB, respectively. In both cases the second-order rate coefficients, $k_{A}$, were found to increase rapidly with aniline concentration, [B], in toluene and the plot of $k_{A} v s$ [B] (Figs. 1 and 2) shows a quadratic dependence, result consistent with a third-order in amine term in the kinetic law. This kinetic behaviour can be interpreted by the mechanism shown in eqs. 1-3 where the dimer $(\mathrm{B}: \mathrm{B})$ of the nucleophile attacks the substrate, $\mathrm{S}$, forming the intermediate, $\mathrm{SB}_{2}$, and a third molecule of amine assisting the decomposition step. The intermediate in eq. 2 is highly zwitterionic and the extra amine molecule help to stabilize the developing charge in the aprotic solvent. The kinetic law is given by eq. 3 where $K=[\mathrm{B}: \mathrm{B}] /[\mathrm{B}] 2$ stands for the amine self association constant. It has been recently shown ${ }^{2,9}$ that for $S_{N} A r$ reactions of nitro-activated substrates and amines in non-polar aprotic solvents, auto-association of amines is very important because of the low permittivity of the media and the importance of hydrogen bonding interactions.

The intervention of the amine on the leaving group departure from the zwitterionic intermediate is the more usual explanation of the autocatalytic behaviour ${ }^{2,9 b}$.

$$
\begin{aligned}
& 2 \mathrm{~B} \stackrel{K}{\rightleftharpoons} \mathrm{B}: \mathrm{B}
\end{aligned}
$$

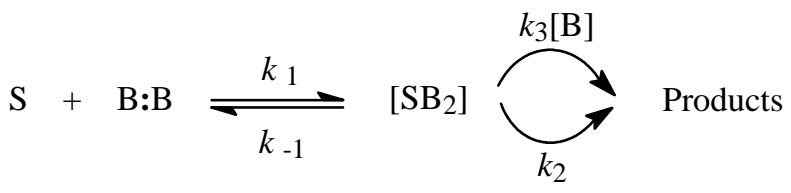




$$
k_{A}=\frac{k_{1} k_{2} K[B]+k_{1} k_{3} K[B]^{2}}{k_{-1}+k_{2}+k_{3}[B]}
$$

On the other hand, the same reaction in acetone $\left(\beta \text { value }=0.48, \mathrm{E}_{\mathrm{T}}(30)=42.2\right)^{11,18}$ and DMSO $\left(\beta\right.$ value $\left.=0.76, \mathrm{E}_{\mathrm{T}}(30)=45.0\right),{ }^{11,18}$ exhibits a linear response of $k_{A} v s[\mathrm{~B}]$, which is characteristic of most base-catalysed reactions by the mechanism shown in eq. 4 . It has been widely observed in $S_{N} A r$ of amines with poor nucleofuges, such fluorine, when the second step is rate determining; the overall second-order rate coefficients obey eq. 5 which comes from the conventional addition-elimination mechanism depicted in eq. 4 for neutral nucleophiles and nitro-activated aromatic substrates. ${ }^{1,2}$

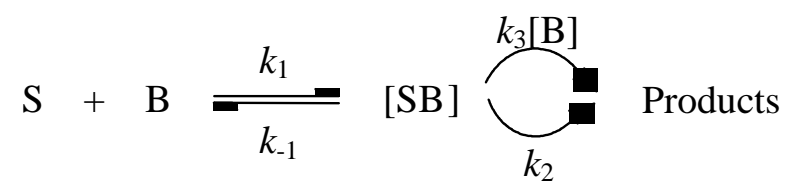

The intercept represents the uncatalysed reaction and the slope measures the base catalysis. When chlorine is the nucleofuge, usually formation of the zwitterionic intermediate, $k_{1}$, is the rate determining step and no base catalysis is observed, but instead a spontaneous decomposition of the intermediate to products is seen, and $k_{A}$ is independent of [B]. ${ }^{1,2}$

$$
k_{A}=\frac{k_{1} k_{2}}{k_{-1}}+\frac{k_{1} k_{3}[B]}{k_{-1}}
$$

In contrast with what would be expected from the widely studied $\mathrm{S}_{\mathrm{N}} \mathrm{Ar}$ with DNClB, the second order rate coefficients, $k_{A}$, of its reactions with aniline exhibit increase with the aniline concentration in the three solvents examined as shown in Figure 1. (Different scales for the $k_{A}$ values should be used in the plots due to the reactivity difference in the three solvents. Standard deviation bars are shown for the reactions in DMSO (regression coef. $r^{2}=0.989$; for reactions in Ac $\mathrm{r}^{2}=0.997$ ). It is interesting to note that while the reactions in dipolar aprotic solvents show a linear dependence with [B], the reactions in toluene exhibits a curvilinear response. While, if $k_{A} /[\mathrm{B}]$ is plotted instead, a linear dependence with [B] is shown, as is typical for the dimer nucleophile mechanism. ${ }^{7}$ With dipolar aprotic solvents of the HBA type as Ac and DMSO, the linear dependence suggest that microscopic aggregation with the solvent competes with the autoaggregation of the nucleophile as shown below. Consistently with this interpretation, although both dipolar aprotic solvent has similar dipolarity/polarity values, the reaction is much faster in DMSO (as shown in Table 1), the solvent that exhibits the greater HBA capacity. ${ }^{11,12}$ In accordance with our proposal of mixed aggregates between aniline and Ac and DMSO, and their 
effect on reaction rates, DMSO has been shown to increase the nitrogen electron density of primary and secondary amines. ${ }^{18,19}$

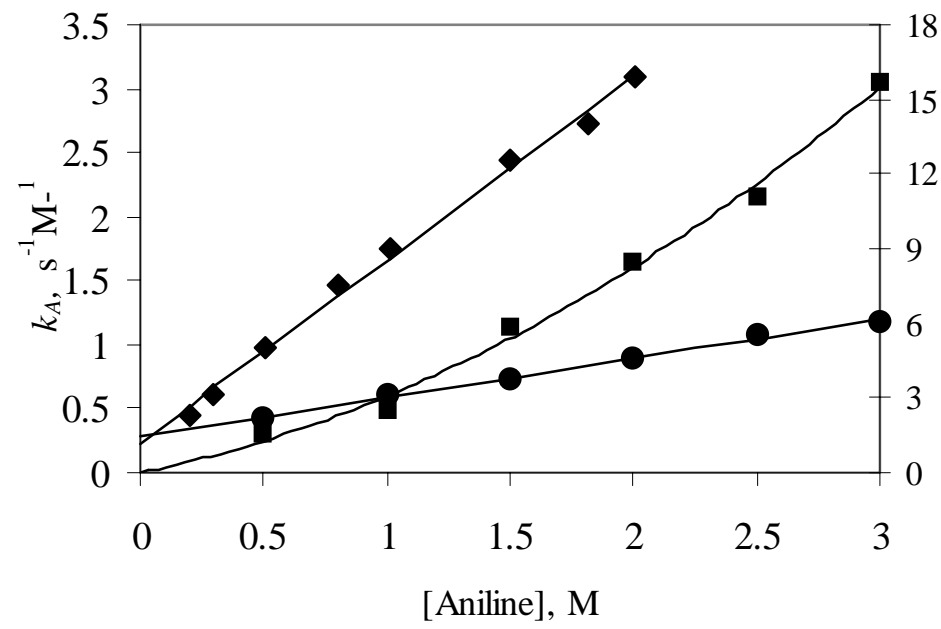

Figure 1. Second-order rate coefficient, $k_{A}$, for the reaction of 2,4-dinitrochlorobenzene, DNClB, with aniline in: DMSO ( $\times 10^{4}$ right $\mathrm{Y}$-axis scale); $\boldsymbol{\square}$ toluene $\left(\times 10^{6}\right.$ left Y-axis scale); acetone $\left(\times 10^{5}\right.$ left $\mathrm{Y}$-axis scale) as a function of [aniline].

Table 1. Reaction of 2,4-dinitrochlorobenzene, DNClB, with aniline, B, in DMSO at $30^{\circ} \mathrm{C}$, toluene and acetone at $40^{\circ} \mathrm{C}$. Second-order, $k_{A}$, rate coefficients

\begin{tabular}{cccccc}
\hline \multicolumn{2}{c}{ DMSO $^{\mathrm{a}}$} & \multicolumn{2}{c}{ Toluene $^{\mathrm{b}}$} & \multicolumn{2}{c}{ Acetone $^{\mathrm{c}}$} \\
\hline [Aniline]/M & $k_{A} \times 10^{4} \mathrm{~s}^{-1} \mathrm{M}^{-1}$ & [Aniline]/M & $k_{A} \times 10^{6} \mathrm{~s}^{-1} \mathrm{M}^{-1}$ & [Aniline]/ $\mathrm{M}$ & $k_{A} \times 10^{5} \mathrm{~s}^{-1} \mathrm{M}^{-1}$ \\
\hline 0.2 & 2.35 & 0.50 & 0.3 & 0.50 & 0.418 \\
0.3 & 3.12 & 1.00 & 0.485 & 1.00 & 0.61 \\
0.51 & 5.00 & 1.50 & 1.14 & 1.50 & 0.788 \\
0.8 & 7.53 & 2.00 & 1.64 & 2.00 & 0.886 \\
1.01 & 9.02 & 2.50 & 2.16 & 2.50 & 1.084 \\
1.5 & 12.51 & 3.00 & 3.05 & 3.00 & 1.174 \\
1.82 & 14.02 & & & & \\
2.01 & 15.95 & & & & \\
\hline
\end{tabular}

${ }^{\mathrm{a}}[\mathrm{DNClB}]=5.0 \times 10^{-4} \mathrm{M}$.

${ }^{\mathrm{b}}[\mathrm{DNClB}]=2.5 \times 10^{-3} \mathrm{M}$; data from ref. $17 \mathrm{~b}$.

${ }^{\mathrm{C}}[\mathrm{DNClB}]=5.08 \times 10^{-4} \mathrm{M}$.

In all the reactions studied in the present work, DNFB reacts faster than DNClB. These results are consistent with those found in bibliography, ${ }^{6 b, 9 b}$ with some exception. Sugiyama and Hayami reported a variable and inverse "Element Effect" in the reactions of picryl halides with 2,4-dimethoxyaniline at low concentration of the nucleophile. ${ }^{10}$ 


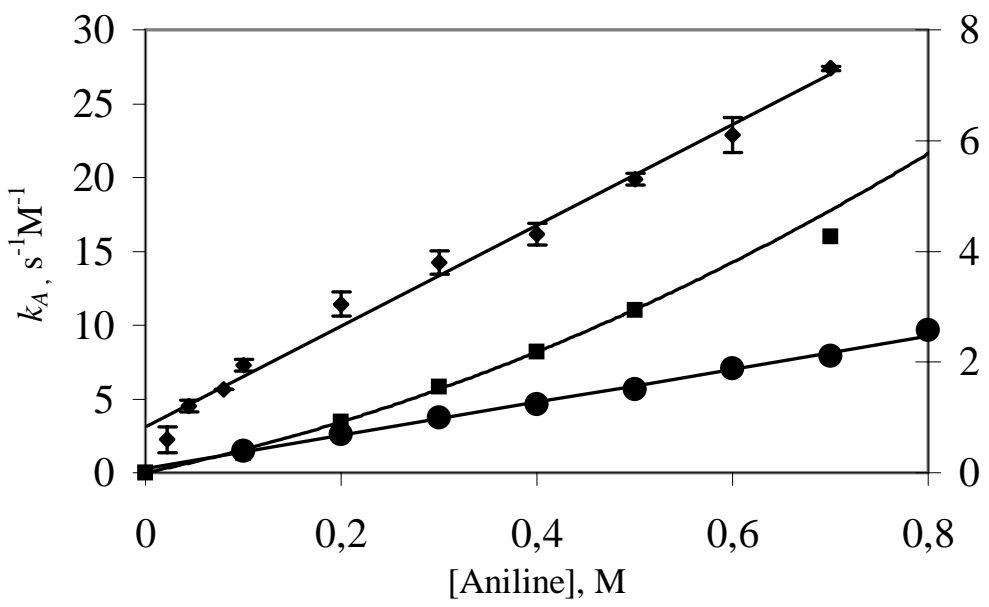

Figure 2. Second-order rate coefficient, $k_{A}$, for the reaction of 2,4-dinitrofluorobenzene, DNFB, with aniline in: DMSO ( $\times 10^{2}$ right $\mathrm{Y}$-axis scale); toluene $\left(\times 10^{6}\right.$ left $\mathrm{Y}$-axis scale); - acetone $\left(\times 10^{4}\right.$ left $\mathrm{Y}$-axis scale) as a function of [aniline].

Table 2. Reaction of 2,4-dinitrofluorobenzene, DNFB, with aniline, B, in toluene, DMSO, and acetone at $30^{\circ} \mathrm{C}$. Second-order, $k_{A}$, rate coefficients

\begin{tabular}{cccccc}
\hline \multicolumn{2}{c}{ Toluene $^{\mathrm{a}, \mathrm{d}}$} & \multicolumn{2}{c}{ DMSO $^{\mathrm{b}}$} & \multicolumn{2}{c}{ Acetone $^{\mathrm{c}}$} \\
\hline [Aniline] $/ \mathrm{M}$ & $k_{A} \times 10^{6} \mathrm{~s}^{-1} \mathrm{M}^{-1}$ & [Aniline] $/ \mathrm{M}$ & $k_{A} \times 10^{2} \mathrm{~s}^{-1} \mathrm{M}^{-1}$ & [Aniline] $/ \mathrm{M}$ & $k_{\mathrm{A}} \times 10^{4} \mathrm{~s}^{-1} \mathrm{M}^{-1}$ \\
\hline 0.1 & 1.32 & 0.022 & 0.60 & 0.1 & 1.05 \\
0.2 & 3.43 & 0.044 & 1.20 & 0.2 & 2.12 \\
0.3 & 5.81 & 0.08 & 1.71 & 0.3 & 3.24 \\
0.4 & 8.22 & 0.1 & 1.95 & 0.4 & 4.32 \\
0.5 & 11.0 & 0.2 & 3.05 & 0.5 & 5.15 \\
0.7 & 16.0 & 0.3 & 3.81 & 0.6 & 6.40 \\
& & 0.4 & 4.31 & 0.7 & 7.78 \\
& & 0.5 & 5.30 & 0.8 & 8.90 \\
& & 0.6 & 6.12 & & \\
\hline
\end{tabular}

${ }^{\mathrm{a}}[\mathrm{DNFB}]=1.73 \times 10^{-4} \mathrm{M}$.

${ }^{\mathrm{b}}[\mathrm{DNFB}]=5.16 \times 10^{-4} \mathrm{M}$.

${ }^{\mathrm{c}}[\mathrm{DNFB}]=5.0 \times 10^{-4} \mathrm{M}$.

${ }^{\mathrm{d}}$ From ref.22.

Similarly to what was observed with DNCIB the reactions are faster in DMSO and Ac, in both solvents the $k_{A}$ dependence with [B] is linear, while it is quadratic in toluene. The same interpretation of different types of nucleophile aggregation applies in this case. To confirm the 
above explanation given for the reactions in pure solvents, the reactions were carried out in acetone-toluene binary solvents.

Reaction of DNCIB and DNFB with aniline in aprotic-dipolar aprotic solvent mixtures. Mixed aggregates formation

The quadratic dependence of $k_{A}$ on [B] observed in aprotic solvents is interpreted within the "dimer nucleophile mechanism" which was previously proposed for other systems considering the strong hydrogen-bond interactions that are expected as needed to stabilize the highly ionic intermediates in the poorly solvating media. ${ }^{7}$ Thus, changes in the reaction media will have a strong influence on the rates and on the kinetics, on the basis of a special effect of a medium hydrogen-bond acceptor co-solvent that competes with the amine itself for aggregation. So, if the "dimer mechanism" applies also in the present case, addition of a hydrogen-bond acceptor (HBA) co-solvent such as acetone to toluene, a non-hydrogen bonding aromatic solvent (NHB), in catalytic amounts should increase the reaction rate by forming a mixed aggregate $\mathrm{RNH}_{2} \ldots \mathrm{OC}(\mathrm{CH} 3)_{2}$, B:Ac, eqn 6, where the amine acts now as a hydrogen-bond donor (HBD) and therefore increases its nucleophilicity, as shown below.
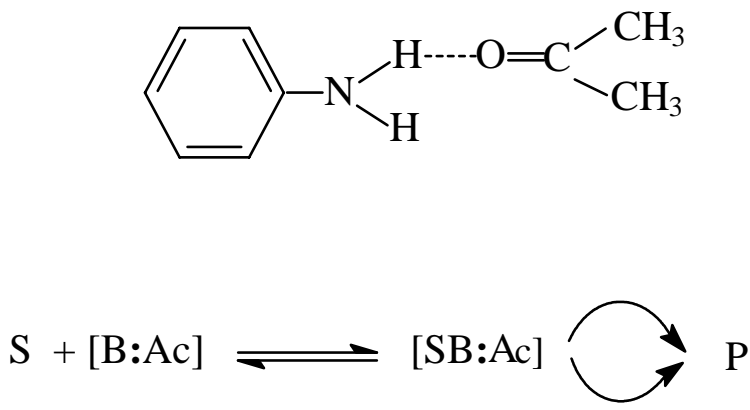

[B]

Table 3. Reaction of 2,4-dinitrochlorobenzene, $\mathrm{DNClB}^{\mathrm{a}}$, with aniline $1 \mathrm{M}$ and $2 \mathrm{M}$, in acetonetoluene binary solvents at $40^{\circ} \mathrm{C}$. Second-order rate coefficients, $k_{A}, 10^{5} \mathrm{~s}^{-1} \mathrm{M}^{-1}$

\begin{tabular}{ccc}
\hline \% Acetone & $k_{A} 10^{5} \mathrm{~s}^{-1} \mathrm{M}^{-1}$ & $k_{A} 10^{5} \mathrm{~s}^{-1} \mathrm{M}^{-1}$ \\
\hline 0 & $1 \mathrm{M}$ & $2 \mathrm{M}$ \\
2 & 0.129 & 0.192 \\
5 & 0.299 & 0.349 \\
7 & 0.348 & 0.385 \\
10 & 0.376 & - \\
20 & 0.425 & 0.447 \\
40 & 0.461 & 0.529 \\
60 & 0.511 & 0.669 \\
80 & 0.564 & 0.767 \\
& 0.613 & 0.836
\end{tabular}




\begin{tabular}{cc}
100 & 0.657 \\
\hline${ }^{\mathrm{a}}[\mathrm{DNClB}]=5 \times 10^{-4} \mathrm{M}$. \\
Table 4 . Reaction of \\
binary solvents at $40^{\circ} \mathrm{C}$. Second-order ratrofluorob \\
\hline \% Acetone & $k_{A} 10^{5} \mathrm{~S}^{-1} \mathrm{M}^{-1}$ \\
\hline & $1 \mathrm{M}$ \\
0 & 0,049 \\
2 & 0,732 \\
5 & 1,250 \\
7 & 1,420 \\
10 & 1,550 \\
20 & 1,690 \\
40 & 1,810 \\
60 & 1,933 \\
80 & 1,990 \\
100 & 2,180 \\
\hline
\end{tabular}

${ }^{\mathrm{a}}[\mathrm{DNFB}]=5 \times 10^{-4} \mathrm{M}$.

Tables 3 and 4 give the observed second-order rate coefficients, $k_{A}$, for the reactions of DNClB and DNFB with aniline in acetone-toluene binary solvents, from small additions of HBA solvent up to pure solvent. Figure 3 and 4 show the important increase in rate observed with small additions of acetone to toluene up to $15 \%$, then the increase with [Ac] decreases. For HBA co-solvent contents $>20 \%$ the reactions show the classical behaviour usually found in basecatalysed $\mathrm{S}_{\mathrm{N}} \mathrm{Ar}^{12,20}$.

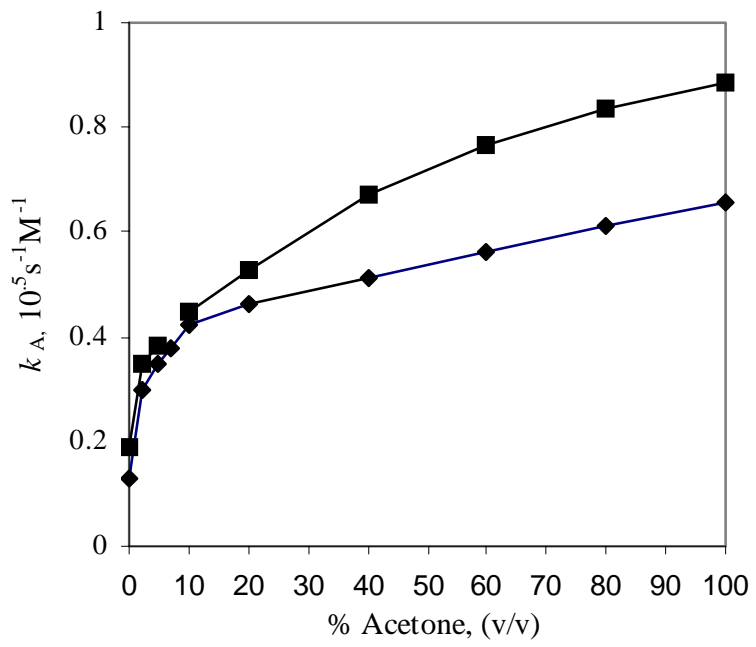


Figure 3. Second-order rate coefficient, $k_{A}$, for the reaction of 2,4-dinitrochlorobenzene, DNClB, with aniline $1 \mathrm{M}, \mathbf{\square} \mathrm{M}$, in acetone-toluene binary solvents as a function of percentage of acetone in the binary solvent, at $40^{\circ} \mathrm{C}$.

Taking into account the increasing effects of small additions of Ac a specific effect must be involved. The specific solvent effects observed for small additions of the HBA co-solvent are consistent with the formation of mixed aggregates nucleophile : co-solvent. Very interestingly, the kinetic behaviour observed for additions of small amounts of a HBA co-solvent was very similar in the reactions of $\mathrm{DNClB}$, a substrate where the first step is considered to be ratedetermining, and in the reactions of DNFB, for which decomposition of the zwitterionic intermediate uses to be the slow step. All these results are consistent with a common interpretation of mixed aggregates in both cases, that competes with the self-aggregation of the nucleophile observed in toluene. The recently reported retarding effects of HBD observed in the reactions of 1-fluoro-2,6-dinitrobenzene with homopiperidine in binary solvent mixtures, ${ }^{5 a}$ are also well accommodated within the overall dimer nucleophile mechanism proposed.

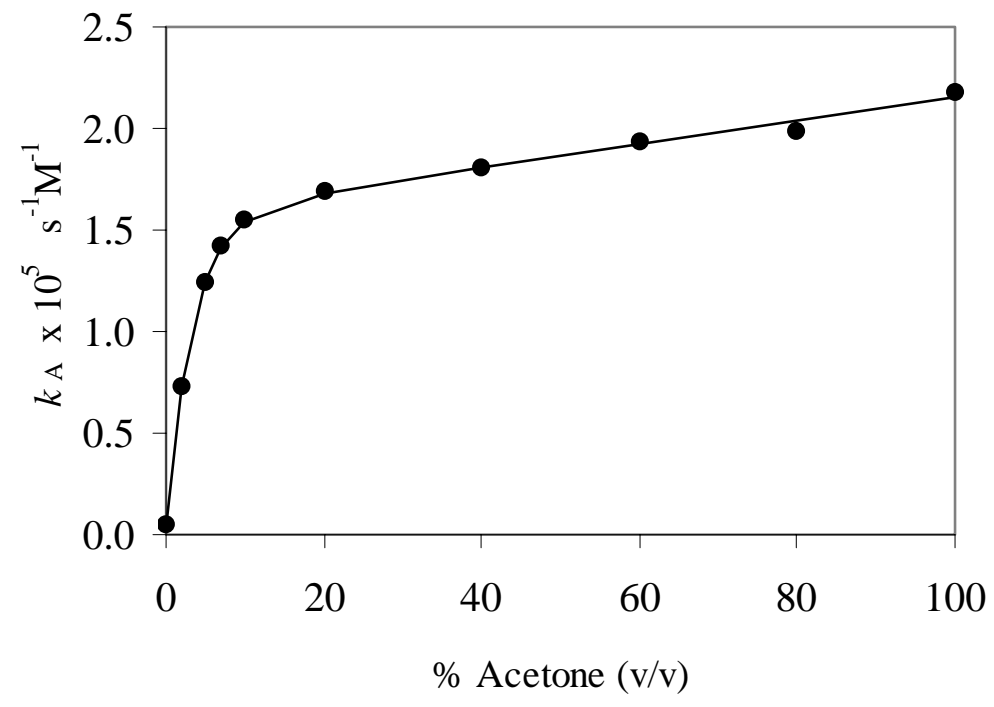

Figure 4. Second-order rate coefficient, $k_{A}$, for the reaction of 2,4-dinitrofluorobenzene, DNFB, with aniline $1 \mathrm{M}$, in acetone-toluene binary solvents as a function of percentage of acetone in the binary solvent, at $40^{\circ} \mathrm{C}$.

Although the catalytic effect of the aggregation of the nucleophile with Ac could also operate in the second step, the above interpretation is preferred since it also explains the early reported "anomalous" catalytic effect of small additions of DMSO observed when the first step is rate determining. $^{21}$. Similar rate accelerations due to the addition of small amounts of a HBA cosolvent were found in the reactions of 1,2-dinitrobenzene with butylamine in benzene. While the reaction is almost insensitive to other additives, the accelerations observed upon addition of 
DMSO to benzene exceed expectations based only on considerations of the polarity of the medium, ${ }^{8,17}$ as the present finding for the reactions studied in toluene:Ac binary solvents.

\section{Conclusions}

Suitable systems to study amine aggregation were examined in nonpolar aprotic and in dipolar aprotic solvents. The reactions of DNClB with aniline in toluene, that should be expected to be independent of the amine concentration, was found to exhibit a rate dependence that is thirdorder in amine consistent with aggregates of the amine acting as the nucleophile. According with this interpretation, the reactions carried out in HBA dipolar aprotic solvents, such as acetone and DMSO, which could form mixed aggregates, show a linear dependence with [B]. The mixed aggregates compete with the amine itself for aggregation and are more basic than the pure dimer.

To confirm the above interpretation the reactions were then studied in binary mixtures acetone:toluene, starting from small amounts of the HBA co-solvent. The great increase in rate observed by small additions of acetone confirmed the formation of mixed aggregates of the nucleophile with the hydrogen-bond acceptor co-solvent, that competes with the amine itself for aggregation and are more basic than the pure dimer. The reactions of DNFB, in which the second-step should be rate-determining, show similar results. All these results confirm that association of amines in aprotic solvents plays an important role in defining the mechanisms of aromatic nucleophilic substitution.

\section{Experimental Section}

\section{Reagents and solvents}

Aniline (Fluka) was kept overnight over potassium hydroxide, distilled over zinc powder and then over sodium; both distillations were carried out under nitrogen at reduced pressure. It was kept in a desiccator protected from light. 2,4-dinitrochlorobenzene (Sigma) was crystallized twice from absolute ethanol $\left(\mathrm{mp} 52-53^{\circ} \mathrm{C} \text {, lit. } 52-53^{\circ} \mathrm{C}\right)^{4 \mathrm{a}}$. 2,4-dinitrofluorbenzene was distilled at reduced pressure under nitrogen, (b.p. $122-123^{\circ} \mathrm{C}$ at $5 \mathrm{mmHg}$, lit. $119^{\circ} \mathrm{C}$ at $2 \mathrm{mmHg}$ ), ${ }^{22} \mathrm{~N}$ (2,4-Dinitrophenyl)aniline (mp $155-156^{\circ} \mathrm{C}$, lit. $\left.155-156{ }^{\circ} \mathrm{C}\right)^{1}$, was prepared from 2,4dinitrochlorobenzene and aniline following the procedure reported for $\mathrm{N}$-(2,4-dinitrophenyl)-2methoxyaniline. Acetone was heated under reflux with successive quantities of potassium permanganate until the violet colour persisted. It was then dried with anhydrous potassium carbonate, filtered from the desiccant and distilled. ${ }^{23}$ Dimethylsulphoxide was dried by standing overnight over calcium hydride. The filtered solvent was then fractionally distilled over calcium hydride under reduced pressure and stored over a Type 4A molecular sieve. ${ }^{23}$ Toluene was kept over sodium wire for several days, distilled twice over sodium. ${ }^{17 \mathrm{~b}}$ Solvents were stored in special vessels protected from light which allow delivery without air contamination. 


\section{Ancillary spectrophotometric measurements}

UV-VIS spectra of the substrates, the product, and different mixtures of both compounds with aniline in the three solvents at several concentrations were recorded in a Shimadzu UV-VIS 240 spectrophotometer. UV spectra of control solutions of the substitution product in toluene, acetone and DMSO containing different amounts of aniline were recorded, and the extinction coefficients determined at $\lambda=450 \mathrm{~nm}$ where the reagents are transparent under these conditions. All the solutions were found to obey Beer's law.

\section{Kinetic procedures}

Kinetics runs were performed by the methods previously reported, ${ }^{24}$ following the appearance of the reaction product at $\lambda=450 \mathrm{~nm}$. The reactions with DNClB were carried out in sealed ampoules (under nitrogen) at $40^{\circ} \mathrm{C}$ and the absorbances were measured in a Shimadzu UV-VIS 240 spectrophotometer. The reactions with DNFB were followed directly in the thermostatted cell of the spectrophotometer at $30^{\circ} \mathrm{C}$. The absorption spectrum of the reaction mixture at "infinite time" corresponded within $\pm 2 \%$ with the "theoretical" value calculated from application of Beer's law to solutions of the product independently prepared in the desired solvent. In all cases pseudo-first-order kinetics were observed. Pseudo-first-order coefficients,

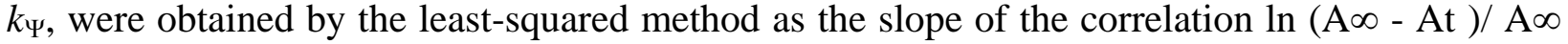
against time, where $\mathrm{A} \infty$ is the optical density of the reaction mixture measured at "infinity" (more than ten half-lives), the second-order rate coefficients, $k_{A}$, were obtained by dividing $k_{\Psi}$ by the amine concentrations. For reactions in mixed solvents, the HBA solvent content $(\mathrm{v} / \mathrm{v})$ refers to its final volume in the reaction mixture. Rate coefficients were reproducible to $\pm 2 \%$. No corrections for expansion coefficients were applied to the concentration values.

\section{Acknowledgements}

NSN is indebted to the hospitality of the Institute for Materials Chemistry and Engineering of Kyushu University, Fukuoka (Japan) where this paper was finished. Financial support from the Universidad Nacional del Comahue (project I-094), Universidad de Buenos Aires and the National Research Council (CONICET) of Argentina is gratefully acknowledged.

\section{References}

1. Terrier, F. "Nucleophile Aromatic Displacement: The influence of the nitro group", In Organic Nitro Chemistry Series; Ferrer, H. Ed.VCH Publishers: New York, 1991.

2. Nudelman, N. S. In The Chemistry of Amino, Nitroso, Nitro and Related Groups, Ed. S. Patai, J. Wiley \& Sons Ltd: London, 1996, Ch. 29. 
3. Crampton, M. In Organic Reaction Mechanisms C. Knipe, W. E. Watts, Eds.; Wiley: Chichester, 1966, Ch. 5A.

4. (a) Nudelman, N. S.; Savini, M.; Alvaro, C. E. S.; Nicotra, V.; Yankelevich, J. S. J.Chem. Soc., Perkin Trans 2 1999, 1627. (b) Nudelman, N. S.; Alvaro, C. E. S.; Savini, M.; Nicotra, V.; Yankelevich, J. S. Collect. Czech. Chem. Commun. 1999, 64, 1583.

5. (a) Mancini, P. M. E.; Fortunato, G.; Adam, C.; Vottero, L. R.; Terenzani, A. J. J. Phys. Org. Chem. 2002, 15, 258. (b) Mancini, P. M. E.; Adam, C.; Perez, Perez, A del C.; Vottero, L. R. J. Phys. Org. Chem. 2000, 13, 221.

6. (a) Boga, C.; Forlani, L. J. Chem. Soc., Perkin Trans 2 2001, 1408. (b) Forlani, L.; Boga, C.; Forconi, M. J. Chem. Soc., Perkin Trans 2 1999, 1455 and references cited therein.

7. Nudelman, N. S. J. Phys. Org. Chem. 1989, 2, 1.

8. Chiacchiera, S. M; Singh, J. O; Anunziata, J. D and. Silver, J. J. J. Chem. Soc., Perkin Trans 2 1988, 1585.

9. (a) Forlani, L. In The Chemistry of Amino, Nitroso, Nitro and Related Groups, Ch. 10, Ed. S. Patai, J. Wiley \& Sons Ltd, London, 1996. (b) Forlani, L. J. Phys. Org. Chem. 1999, 12, 417.

10. Sugiyama N.; Hayami, J. Chem. Soc. of Japan 1999, 691.

11. Reichardt, C. Chem. Rev. 1994, 94, 2319.

12. Abboud, J. L. M.; Notario, R. Pure Appl. Chem. 1999, 71, 645.

13. (a) Marcus, Y. J. Solution Chem. 1991, 20, 929. (b) Chem. Soc. Rev. 1993, 409.

14. (a) Mancini, P. M. E.; Terenzani, A.; Gasparri, M. G.; Vottero, L. R. J. Phys. Org. Chem. 1995, 8, 617. (b) Mancini, P. M. E.; Terenzani, A.; Adam, C.; Vottero, L. R, J. Phys. Org. Chem. 1999, 12, 430. (c) Mancini, P. M. E.; Terenzani, A.; Adam, C.; Perez, A del C.; Vottero, L. R. J. Phys. Org. Chem. 1999, 12, 713.

15. Nagy, O. A. Bull. Soc. Chim. Belg. 1985, 94, 11.

16. Nudelman, N. S.; Palleros, D. J. Org. Chem. 1983, 48, 1613.

17. (a) Nudelman, N. S.; Marder, M.; Gurevich, A. J. Chem. Soc., Perkin Trans 2 1993, 229. (b) Nudelman, N. S.; Alvaro, C. E. S.; Yankelevich, J. S. J .Chem. Soc., Perkin Trans. 2 1997, 2125.

18. Mancini, P. M. E.; Terenzani, A.; Adam, C.; Vottero, L. R. J. Phys. Org. Chem. 1997, 10, 849.

19. Crampton, M.; Robotham, I. A. J. Chem. Research (S) 1997.

20. Palleros, D.; Nudelman, N. S. J .Chem. Soc., Perkin Trans 2 1985, 479.

21. Suhr, H. Ber. Bunsenges. Phys. Chem. 1963, 67, 893.

22. Nudelman N. S.; Montserrat, J. M. J.Chem. Soc., Perkin Trans 2 1990, 1073.

23. Vogel, A. Practical Organic Chemistry; $5^{\text {th }}$ Edn Longman: London, 1991.

24. Bunnet, J. F.; Kato, T.; Nudelman, N. S. In Fundamental Organic Chemistry Laboratory Manual, Eds. Finley, K. T.; Wilson, J. Prentice-Hall: New Jersey, 1974; p 112. 